\title{
Functions of $\mathrm{CaPhm} 7$ in the regulation of ion homeostasis, drug tolerance, filamentation and virulence in Candida albicans
}

\author{
Linghuo Jiang ${ }^{*}$ (D) and Hongbo Pan
}

\begin{abstract}
Background: Calcium-permeable transient receptor potential (TRP) channels exist in eukaryotic cells from yeasts to animals and plants. and they act as sensors for various stresses. Arabidopsis thaliana calcium permeable stress-gated cation channel 1 (AtCSC1) was the first plant calcium-permeable TRP to be describe $\bar{d}$ and can be activated by hyperosmotic shock. Candida albicans CaPHM7 is one of the sequence homologs of AtCSC1, but its function remains unknown.

Results: We show here that CaPhm7 is localized to the plasma membrane in both the yeast and hyphal cells of $C$. albicans. C. albicans cells lacking CaPHM7 are sensitive to SDS and ketoconazole but tolerant to rapamycin and zinc. In addition, deletion of CaPHM7 leads to a filamentation defect, reduced colony growth and attenuated virulence in the mouse model of systemic infection.

Conclusions: CaPhm7 is involved in the regulation of ion homeostasis, drug tolerance, filamentation and virulence in this important human fungal pathogen. CaPhm7 could be a potential target of antifungal drugs.
\end{abstract}

Keywords: Anoctamin, TMEM16, Calcium permeable stress-gated cation channel, Phm7, CSC1, Ion

\section{Background}

Calcium homeostasis and calcium signaling are highly conserved during evolution in eukaryotic cells $[1,2]$. The cytosolic calcium concentration is maintained by calcium channels, pumps, exchangers and other regulators in the plasma membrane and intracellular organelle membranes of eukaryotic cells [2-4]. Elevation of the cytosolic calcium concentration is one of the earliest responses to stress in eukaryotic cells [5, 6]. Calcium-permeable transient receptor potential (TRP) channels exist in animals, plants and yeasts, and act as sensors for various stresses, including temperature, $\mathrm{pH}$, osmolarity and nutrient availability [713]. The first calcium-permeable TRP, initially isolated from Arabidopsis thaliana, can be activated by hyperosmotic shock and, therefore, is named as calcium permeable

\footnotetext{
* Correspondence: linghuojiang@sdut.edu.cn

Laboratory for Yeast Molecular and Cell Biology, the Research Center of Fermentation Technology, School of Agricultural Engineering and Food Science, Shandong University of Technology, Zibo 255000, China
}

stress-gated cation channel 1 (CSC1) [8]. AtCSC1 was also independently isolated through a genetic approach and named as reduced hyperosmolarity-induced $\left[\mathrm{Ca}^{2+}\right]_{\mathrm{i}}$ increase 1 (OSCA1) [7]. AtCSC1 and its Saccharomyces cerevisiae homolog ScCSC1 also belong to the anoctamin/ TMEM16 family [8].

The anoctamin/TMEM16 family members function in many physiological processes, including ion homeostasis and phospholipid scrambling [14-16]. TMEM16A (also known as ANO1) and TMEM16B (ANO2) form the $\mathrm{Ca}^{2+}$-activated $\mathrm{Cl}^{-}$channel, which is important for transepithelial ion transport, olfaction, phototransduction, smooth muscle contraction, nociception, cell proliferation and control of neuronal excitability [14]. The $\mathrm{Ca}^{+}$-activated $\mathrm{Cl}^{-}$channel formed by $\mathrm{CaCC}$ and TMEM16F regulates phospholipid scramblase [15]. TMEM16F itself also functions as a phospholipid scramblase [16]. However, functions of other family members remain to be elucidated. Mutations in four

(c) The Author(s). 2018 Open Access This article is distributed under the terms of the Creative Commons Attribution 4.0 International License (http://creativecommons.org/licenses/by/4.0/), which permits unrestricted use, distribution, and reproduction in any medium, provided you give appropriate credit to the original author(s) and the source, provide a link to the Creative Commons license, and indicate if changes were made. The Creative Commons Public Domain Dedication waiver (http://creativecommons.org/publicdomain/zero/1.0/) applies to the data made available in this article, unless otherwise stated. 
anoctamin genes (ANO3, ANO5, ANO6, and ANO10) cause various genetic diseases. For example, mutations in the plasma membrane protein TMEM16F impair $\mathrm{Ca}^{2+}$-dependent externalization of phosphatidylserine in activated platelets, erythrocytes and lymphocytes, which leads to Scott syndrome, a bleeding disorder $[15,16]$. In addition, a few other anoctamin-related diseases have been identified, such as ataxia and dystonia, persistent borrelia and mycobacteria infection, skeletal syndromes, gnathodiaphyseal dysplasia as well as cancers [17]. TMEM16C (ANO3) is associated with childhood mumps and rubella vaccination-related febrile seizures [18].

Candida albicans is the most common opportunistic human fungal pathogen, which causes life-threatening systemic infections in immunocompromised patients [19, 20]. To explore the biological functions of $C$. albicans homologs of CSC1 and other anoctamin/TMEM16 family members, using the amino acid sequence of AtCsc1 as a query, we blasted the C. albicans genome and revealed five AtCsc1 homologous proteins, CaPhm7, CaRsn1, CaSpo75, orf19.1823 (C1_06270W) and orf19.5932 (C3_04720C). CaPhm7 is a protein of 927 amino acids, which shares $21 \%$ (42\%), 36\% (59\%), 26\% (44\%), 20\% (41\%) and 21\% (42\%) identities (similarities) with AtCsc1, CaRsn1, CaSpo75, C1_06270Wp and C3_04720Cp, respectively, in amino acid sequences (Additional file 1: Figure S1). In this study, we have characterized the biological functions of CaPHM7 in C. albicans.

\section{Methods}

\section{Strains, media, plasmids and primers}

C. albicans strains and plasmids used in this study are listed in Tables 1 and 2, respectively. Primers used in this study are listed in Additional file 1: Table S1. C. albicans cells were maintained in YPD medium (2\% glucose, $2 \%$ peptone, $1 \%$ yeast extract) or SD medium $(0.67 \%$ yeast nitrogen base without amino acids, $2 \%$ glucose, and auxotrophic amino acids as needed) at $30{ }^{\circ} \mathrm{C}$. Antifungal drugs were obtained from Sigma.

Table 1 Strains used in this study

\begin{tabular}{lll}
\hline Strain & Genotype & Source \\
\hline CAl4 & ura3::Aimm434/ura3::Aimm434 & {$[38]$} \\
PHBCA57 & CAl4 PHM7/phm7::natR & This study \\
PHBCA58 & CAl4 PHM7/phm7::frt & This study \\
PHBCA62 & CAl4 phm7::frt/phm7::natR & This study \\
XDCA06 & CAl4/Clp10 & {$[32]$} \\
PHBCA100 & CAl4 PHM7/phm7::frt/Clp10 & This study \\
PHBCA102 & CAl4 phm7::frt/phm7::frt/Clp10 & This study \\
PHBCA105 & CAl4 phm7::frt/phm7::frt/Clp10-CaPHM7 & This study \\
PHBCA107 & CAl4 PHM7/PHM7::GFP-URA3 & This study \\
PHBCA126 & CAl4 PHM7::GFP-URA3/phm7::natR & This study \\
\hline
\end{tabular}

Table 2 Plasmids used in this study

\begin{tabular}{lll}
\hline Plasmid & Description & Source \\
\hline Clp10 & $\begin{array}{l}\text { C. albicans integration vector } \\
\text { with URA3 marker }\end{array}$ & {$[30]$} \\
Clp10-CaPHM7 & $\begin{array}{l}\text { Full-length CaPHM7 gene in } \\
\text { Clp10 }\end{array}$ & This study \\
pSFS2 & The SAT1 flipper cassette & {$[23]$} \\
pGFP-URA3 & C-terminal GFP integration & {$[24]$} \\
& with URA3 marker & \\
\hline
\end{tabular}

\section{DNA manipulation}

There is one PstI site (CTGCAG), which contains a codon (bolded), encoding the A499 residue of $\mathrm{CaPhm} 7$, in both alleles A and B of the CaPHM7 gene. However, due to the presence of a synonymous coding single nucleotide polymorphism (GCA $\rightarrow$ GCT) (cSNP) between alleles A and B of the CaPHM7 gene encoding the A705 residue of $\mathrm{CaPhm} 7$, there is an additional PstI site (CTGCAG) overlapping with this cSNP site in the allele A of CaPHM7. Therefore, using the primer pair pRS316-CaPHM7-F/ CaPHM7 TY-UP-R, we first amplified the DNA fragment containing the 739-bp promoter and the upstream 1498-bp ORF region of CaPHM7, cut it with HindIII and PstI, and cloned it into the CIp10 vector, yielding CIp10-CaPHM7UP. Second, using the primer pair CaPHM7 TY-DOWN-F/CaPHM7 TY-DOWN-R, from the allele B of CaPHM7 we amplified a 1.58-kb DNA fragment containing the downstream 1307-bp ORF region and the 321-bp terminator region of CaPHM7, cut with PstI, and clone the 1.58-kb DNA fragment into the Pst $\mathrm{I}$ site of CIp10-CaPHM7UP, generating CIp10-CaPHM7. These inserts were sequenced to ensure no mutation and correct orientation.

\section{Disruption of two alleles for CaPHM7}

To delete the first allele of CaPHM7 in the CAI4 background, we PCR amplified the SAT1 flipper cassette with primers CaPHM7-NAT-UP and CaPHM7-NAT-DOWN from the plasmid pSFS2 [21-23], and transformed it into the CAI4 strain. The resulting PHBCA57 strain (PHM7/ phm7::natR) was grown in YPD medium containing $25 \mu \mathrm{g} \mathrm{ml}^{-1}$ nourseothricin for FLP-mediated excision of the SAT1 cassette, which generated PHBCA58 strain (PHM7/phm7::frt). Similarly, we disrupted the second allele, which generated the PHBCA62 strain (phm7::frt) phm7::natR) (Additional file 1: Figure S2).

\section{Chromosomal tagging of $\mathrm{CaPhm7}$ with green fluorescent protein (GFP)}

To generate a C-terminal GFP-tagged CaPHM7-GFP fusion protein, from the plasmid pGFP-URA3 [24], we used the primers CaPHM7-GFP-UP and CaPHM7-GFP-DOWN to amplify the GFP-URA3 cassette flanked with 100-bp CaPHM7 DNA fragments. This cassette was transformed 
into both the wild type CAI4 and the heterozygous mutant PHBCA57. Genotypes of the resulted CaPHM7-GFP strains PHBCA107 and PHBCA126 were verified by PCR with primer pairs CaPHM7-GFP-F/CaPHM7-GFP-R and GFP-R/CaURA3-F, respectively (Data not shown).

\section{Phenotype tests}

Test strains were grown overnight in liquid medium at $30{ }^{\circ} \mathrm{C}$, serially diluted by 10 -fold and spotted sequentially onto plates as described previously $[25,26]$. Plates were photographed after they were incubated for 2-3 days at $30{ }^{\circ} \mathrm{C}$. Filamentation was assessed by inoculating cells with an appropriate dilution into various filamentation-induction liquid media, and cells were grown with shaking at $37{ }^{\circ} \mathrm{C}$. For colony morphology observation 20 cells from each strain was plated onto YPD 10\% serum, Lee's and Spider medium [27, 28], and incubated for $5-7$ days at $37{ }^{\circ} \mathrm{C}$. These experiments were repeated at least three times.

\section{Growth curve assay}

Cells of the wild type CAI 4 + CIp10, the heterozygous mutant PHM7/phm7+CIp10, the homozygous mutant phm7/phm7+CIp10 and the revertant phm7/phm7+ CIp10-CaPHM7 were cultured overnight in SD-URA medium at $30{ }^{\circ} \mathrm{C}$, serially diluted for 10 times in fresh YPD medium and grown further for $10 \mathrm{~h}$. Culture samples of each strain were taken every $2 \mathrm{~h}$ for $\mathrm{OD}_{600 \mathrm{~nm}}$ measurement. Data were the average of three independent experiments and analyzed by GraphPad Prism software. $P$ values of $<0.05$ were considered to be significant.

\section{Virulence assays}

Strains tested for virulence were integrated with the plasmid CIp10, so that URA3 was expressed from the neutral RPS1 locus [29, 30]. Phenotypes of all CIp10-integrated mutants were confirmed (Data not shown). Male BALB/c mice of 8 weeks old and 25-30 g (Geruisiwei Inc., Suzhou, China) were maintained in individually ventilated cages. Cell suspension at a concentration of $1 \times 10^{7}$ cells ml $^{-1}$ in $0.9 \%(w / v) \mathrm{NaCl}$ solution was prepared for each strain. Twelve mice for each strain were inoculated with $0.1 \mathrm{ml}$ of cell suspension per mouse via a lateral tail vein. Mouse survival rates were monitored daily. If mice appeared to be curled up, hair fluffy, dull and not responsive to outside stimuli, they were sacrificed through cervical vertebra. Survival curves were generated according to the Kaplan-Meier method using the PRISM program (GraphPad Software) and compared using the log-rank test.

After $48 \mathrm{~h}$ of inoculation, two mice were randomly executed, and the colony forming unit (CFU) in their kidneys was analyzed on SD-URA plates as described [31, 32]. Stained sections of kidney tissues were examined for fungal infiltration. Mouse studies were carried out in accordance with the guidelines established by the Ethics Committee of Nantong University, China.

\section{Results \\ Deletion of CaPHM7 causes C. albicans cells sensitive to SDS and ketoconazole and tolerant to rapamycin and zinc ion}

Unlike AtCsc1 with nine TM domains, CaPhm7 has 11 TM domains (Additional file 1: Figure S1). However, $\mathrm{CaPhm} 7$ is highly conserved in ascomycetes including other human pathogenic Candida species, and shares $94 \%$ (98\%), 42\% (64\%), 82\% (91\%), 43\% (64\%) and 46\% (67\%) identity (similarity), respectively, with $C$. dubliniensis CdPhm7, C. glabrata CgPhm7, C. tropicalis CtPhm7, S. cerevisiae ScPhm7 and Schizosaccharomyces pombe SpPhm7 (Additional file 1: Figure S3). To understand the biological functions of $\mathrm{CaPhm} 7$ in C. albicans, we disrupted the two alleles of CaPHM7 (Table 1; Additional file 1: Figure S2). As compared to the wild type, the homozygous mutant was sensitive to SDS and tolerant to rapamycin (Fig. 1a). In contrast, both the heterozygous mutant and the homozygous mutant were sensitive to ketoconazole (KCZ) and tolerant to $\mathrm{ZnCl}_{2}$ (Fig. 1b). However, no alternation was observed in the sensitivity of these mutants to other agents such as calcium $(0.6 \mathrm{M})$, manganese $(1 \mathrm{mM})$, magnesium $(10 \mathrm{mM})$, sodium $(1.5 \mathrm{M})$, potassium $(1.5 \mathrm{M})$, lithium and cadmium $(100 \mu \mathrm{M})$ ions (Data not shown). The SDS-sensitive, rapamycin-tolerant and zinc-tolerant phenotypes of the homozygous mutant could be reversed by introducing one allele of the CaPHM7 gene (Fig. 1). However, it should be noted that reintroduction of CaPHM7 only partially restored growth of the homozygous mutant on ketoconazole. On zinc, the revertant (one copy of CaPHM7) restored the wild type phenotype, but the heterozygous mutant grew at levels similar to the homozygous mutant (Fig. 1). Furthermore, the colonies of both the homozygous mutant and the revertant were slightly smaller than those of the wild type on solid YPD plates (Fig. 1). However, the growth rates of both the homozygous mutant and the revertant were not significantly different from the wild type (Additional file 1: Figure S4).

\section{$\mathrm{CaPhm7}$ is localized to the plasma membrane}

To examine the subcellular localization of $\mathrm{CaPhm} 7$ proteins, we integrated the GFP tag at the C-terminus of the $\mathrm{CaPHM} 7$ allele in the wild type CAI4 and the heterozygous mutant, which generated PHBCA107 and PHBCA126 strains, respectively. Both showed similar phenotypes to the wild type CAI4 on plates containing SDS, ketoconazole, rapamycin or $\mathrm{ZnCl} 2$ (Fig. 2a), indicating the CaPHM7-GFP allele is functional. Western blot analysis indicated that the CaPHM7-GFP expressed as a protein of $126 \mathrm{kDa}$, as expected, in cells of both PHBCA107 and PHBCA126 (Additional file 1: Figure S5). This CaPHM7-GFP protein 


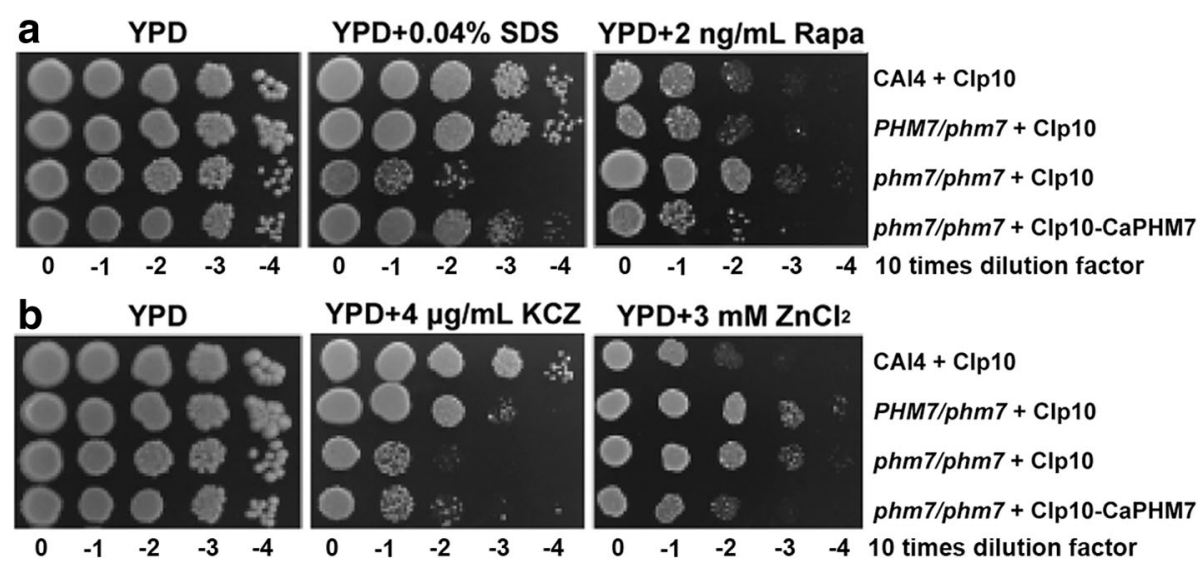

Fig. 1 C. albicans cells lacking CaPHM7 are sensitive to SDS and ketoconazole (KCZ) (a) and tolerant to rapamycin and zinc (b). The wild type, the heterozygous mutant and the homozygous mutant, integrated with the Clp10 vector or Clp10-CaPHM7, were grown overnight in SD-URA medium, serially diluted by 10 times and spotted onto YPD plates indicated. Plates were incubated at $30^{\circ} \mathrm{C}$ for $48 \mathrm{~h}$ (a) or $72 \mathrm{~h}$ (b) before photos were taken. KCZ, ketoconazole; Rapa, rapamycin

was localized to the plasma membrane in both the log-phase yeast-form cells and the filamentous cells of the PHBCA107 strain induced in YPD $+10 \%$ FBS, Spider or Lee's media (Fig. 2b). Taken together, these data indicate that $\mathrm{CaPhm} 7$ is a plasma membrane protein.

\section{Deletion of CaPHM7 leads to a defect in filamentation, reduced colony growth and attenuated virulence}

Hyphal development is related to the virulence of $C$. albicans cells [20,33]. To examine the function of CaPHM7 in hyphal development, we performed filamentous growth assays. In liquid YPD plus 10\% FBS, Lee's or Spider media, the homozygous mutant showed a defect in filamentation (Fig. 3a). On these solid media, comparing to the wide type and the heterozygous mutant, the homozygous mutant showed reduced colony sizes on solid YPD plus 10\% FBS, Lee's and Spider media (Fig. 3b). These defects of the homozygous mutant could be complemented by reintroduction of a single copy of the CaPHM7 allele (Fig. 3).

To determine the role of CaPHM7 in C. albicans virulence, we infected mice intravenously with $1 \times 10^{6}$ cells of the wild-type parental control strain and the homozygous mutant. Two mice were randomly selected from each group and sacrificed after $48 \mathrm{~h}$ to make sure that mice had been infected, with the CFUs/g reaching $1.02 \times 10^{7}(n=2)$ in wet kidney tissue of the homozygous mutant, $1.56 \times 10^{7}(n=2)$ in that of the wild-type and $1.38 \times 10^{7}(n=2)$ in that of the complemented strain. By Day 6, none of the mice injected with the wild type survived, while $30 \%$ and $80 \%$ of the mice inoculated with the complemented strain and the homozygous mutant, respectively, survived. By Day 8 , none of the mice inoculated with the complemented strain survived, while the mice injected with the homozygous mutant survived until Day 20 (Fig. 4a). As a control, no hyphal or yeast cells of C. albicans was observed in the kidney tissues of mice inoculated with saline as a control (Fig. 4b). Compared to kidney tissues of mice infected with the wild type and the complemented strain, those infected with the homozygous mutant were less infiltrated by hyphal filaments (Fig. 4b). Taken together, these results suggest that deletion of CaPHM7 attenuates the virulence of $C$. albicans cells in this systemic infection model.

\section{Discussion}

In this study, we have shown that the C. albicans homolog of AtCSC1, CaPhm7, is localized in the plasma membrane of both yeast-form and filamentous cells. C. albicans cells lacking $\mathrm{CaPHM7}$ are sensitive to membrane-disturbing agents, SDS and ketoconazole. AtCSC1 and ScCSC1 ion channels are permeable to various cations, including $\mathrm{Ca}^{2+}$, $\mathrm{K}^{+}$and $\mathrm{Na}^{+}$and can be activated by hyperosmotic shock [8]. However, lack of CaPHM7 leads to a tolerance to $\mathrm{Zn}^{2+}$, but does not affect the sensitivity of $C$. albicans cells to other ions tested, including $\mathrm{Ca}^{2+}, \mathrm{K}^{+}, \mathrm{Na}^{+}, \mathrm{Mg}^{2+}, \mathrm{Mn}^{2+}$, $\mathrm{Cd}^{2+}$, and $\mathrm{Li}^{+}$. C. albicans cells lacking CaPHM7 are also not sensitive to osmotic stresses such as $1 \mathrm{M}$ mannitol and $4 \mathrm{M} \mathrm{KCl}$. Therefore, whether $\mathrm{CaPhm} 7$ is a calcium permeable stress-gated cation channel in the plasma membrane remains to be determined. It is also interesting to note that haploinsufficiency occurs to the deletion of one of the CaPHM7 alleles in the response to ketoconazole and zinc ion, but not to SDS and rapamycin (Fig. 1b). Among anoctamins, ANO10 shows the highest degree of homology to ScIst2, with a $36 \%$ similarity and a $17 \%$ identity in their amino acid sequences [17]. CaPhm7 shares 18\% (34\%) and $14 \%$ (30\%) identity (similarity) with HsAno10 and ScPhm7, respectively, in their amino acid sequences (Additional file 1: Figure S6). This suggests that $\mathrm{CaPhm} 7$ might be a member of the anoctamin/TMEM16 family member. 

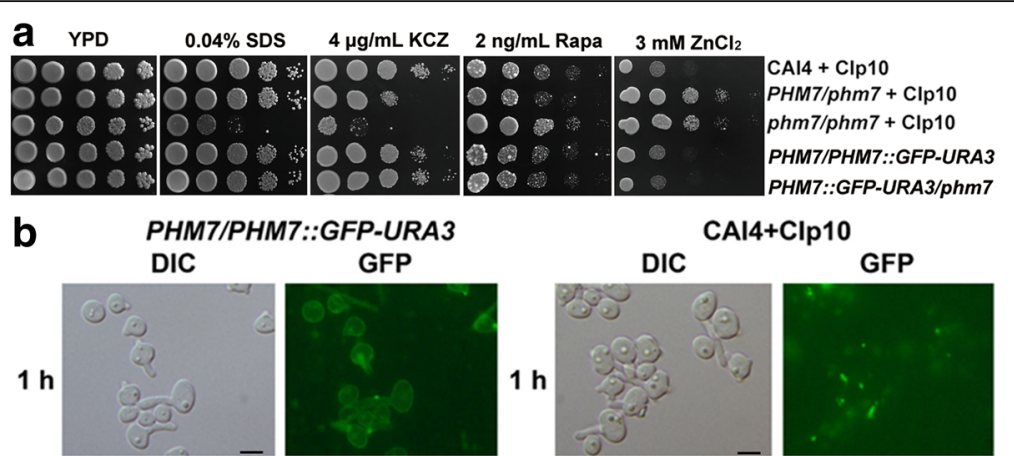

GFP

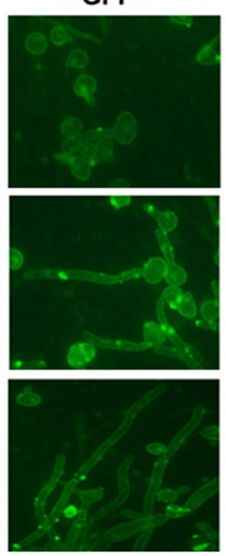

YPD+10\% FBS

PHM7/PHM7::GFP-URA3

DIC
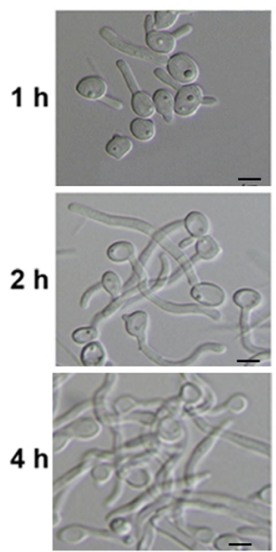

Spider
CAl4+CIp10

DIC
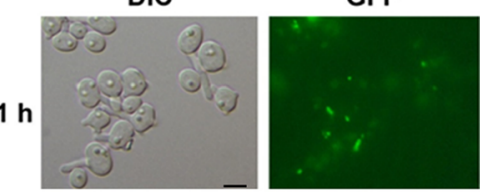

$2 \mathrm{~h}$
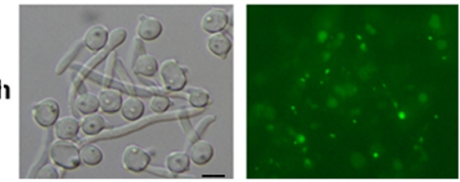

$4 \mathrm{~h}$
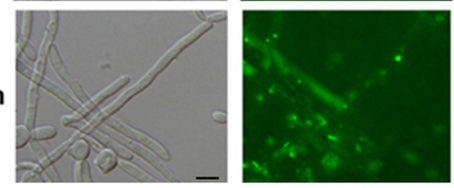

YPD+10\% FBS

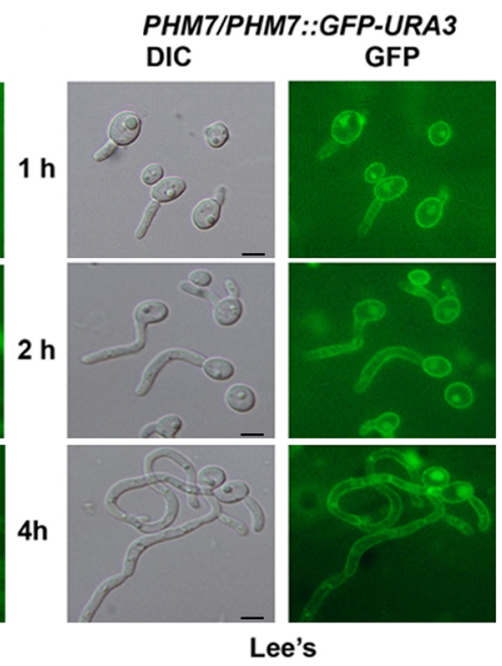

Fig. 2 Subcellular localization and mouse virulence assay for CaPhm7. a Functional assay of the CaPHM7-GFP allele. The wild type CAI4 (XDCA06), the heterozygous mutant PHBCA100, the homozygous mutant PHBCA 102, as well as PHBCA107 and PHBCA126, carrying the CaPHM7-GFP allele, were grown overnight in SD-URA medium, serially diluted by 10 times and spotted onto YPD plates indicated. b Visualization of GFP signals within PHBCA107 cells of log-phase yeast-form and filamentous form induced in YPD + 10\% FBS (top left), Spider (bottom left) or Lee's (bottom right) media at $1000 \times$ magnification on a Nikon ECLIPSE 80i microscope. Visualization of the wild type CAl4 cells (top right) as a control for hyphal morphology and green auto-fluorescence. DIC, differential interference contrast. The scale bars represent $5 \mu \mathrm{M}$

In addition, we have shown that $C$. albicans cells lacking CaPHM7 are tolerant to rapamycin, the specific inhibitor of the target of rapamycin (TOR) kinase, which indicates that the TOR kinase activity might be inhibited in C. albicans cells lacking CaPHM7. This is consistent with our observations that deletion of CaPHM7 leads to a reduced colony growth, which might be related to a reduced activity for the central controller of cell growth, TOR [34].

The S. cerevisiae homolog of CaPHM7, ScPHM7 has initially been identified to be the phosphate metabolism gene
7 and, together with other 21 genes, is regulated by the PHO regulatory pathway in S. cerevisiae [35]. However, the function of ScPHM7 in phosphate metabolism is not defined up to now. ScPhm7 shares a $43 \%$ identity and $64 \%$ similarity, respectively, with $\mathrm{CaPhm} 7$ in amino acid sequence (Additional file 1: Figure S1). Our results on $\mathrm{CaPHM} 7$ suggests that $\mathrm{ScPhm} 7$ might be a plasma membrane CSC ion channel that links the extracellular phosphate availability to the intracellular calcium signaling pathway to regulate phosphate metabolism. Recent studies 

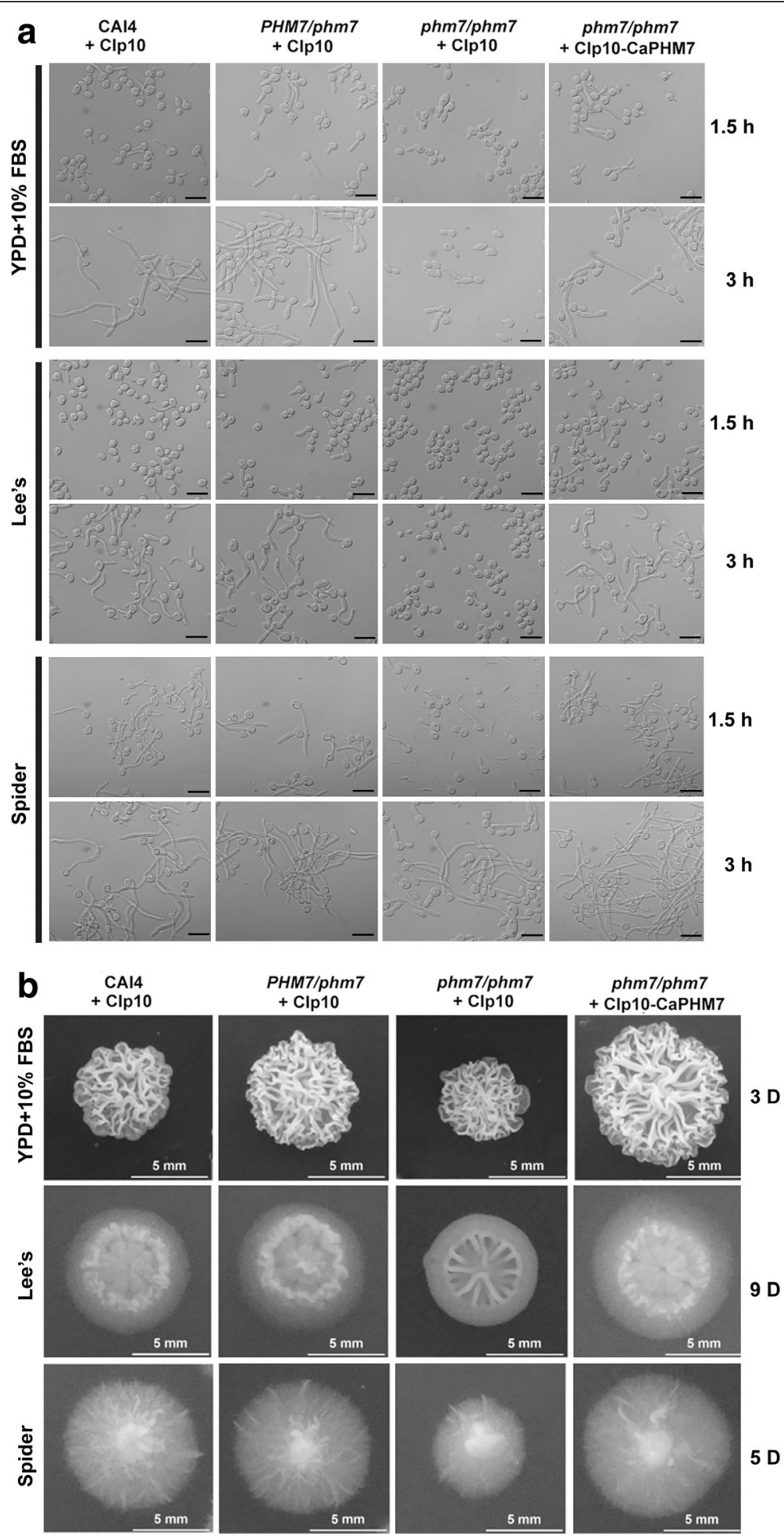

Fig. 3 Functions of CaPHM7 in filamentation and colony formation. The wild type CAl4, the heterozygous mutant and the homozygous mutant, carrying the Clp10 vector or Clp10-CaPHM7, were grown overnight in SD-URA medium, and inoculated to liquid YPD containing 10\% fetal bovine serum (FBS), Lee's or Spider media for filamentation assay (a) or onto their solid plates for colony morphology oberservation (b). Incubation was carried out at $37^{\circ} \mathrm{C}$. The scale bar in (a) represents $10 \mu \mathrm{M}$, while the scale bar in (b) represents $5 \mathrm{~mm}$ 


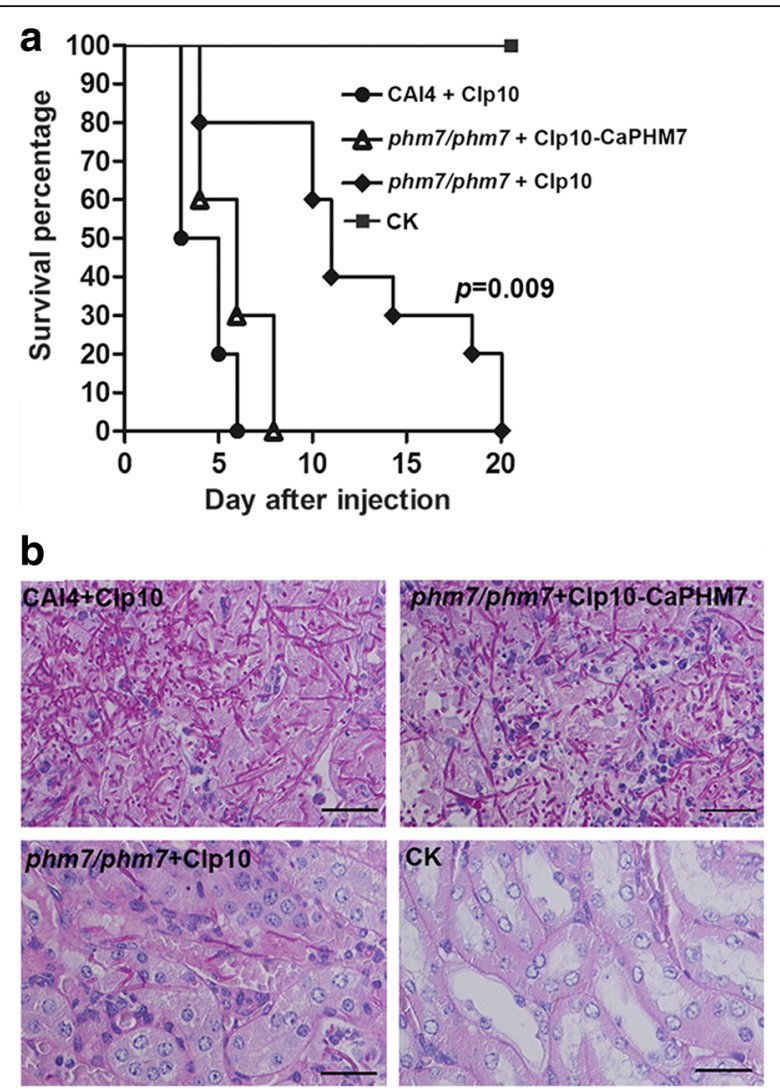

Fig. 4 Virulence assay. a survival rates of mice $(n=10)$ infected with the wide type CAl4, the homozygous phm7/phm7 mutant and its complemented strain. Mice were checked every day for morbidity, and survival was monitored for 30 days. The $p$ value (0.009) of the log-rank test indicates that the survival curves between the wild-type CAI 4 and the phm7/phm7 mutant is significantly different. $\mathbf{b}$ histopathological examination of kidney tissues of moribund mice infected with the wild-type, the homozygous mutant and its complemented strain. Saline buffer was injected as negative control (CK). Infected kidney tissues from mice were stained with periodic acid-Schiff's reagent. Representatives of five kidney cross-sections from two mice per strain were photographed with $40 \times$ lenses. The hyphal cells are highlighted by arrows. The scale bars represent $20 \mu \mathrm{M}$

have indeed linked the calcium signaling pathway to phosphate metabolism. Both the $\mathrm{PHO} 4$ gene, encoding the transcription factor of the PHO pathway, and the PHO89 gene, encoding the high-affinity inorganic phosphate $(\mathrm{Pi})$ transporter, are transcriptionally regulated by the calcium/ calcineurin signaling in S. cerevisiae [36]. Calcineurin is also involved in the regulation of the PHO pathway in $A s$ pergillus fumigatus [37]. Therefore, like AtScs1 as an osmolarity sensor in the plant $A$. thaliana $[7,8], \mathrm{ScPhm} 7$ might function as a sensor for extracellular phosphate levels in S. cerevisiae.

In this study, we have observed that the homozygous mutant for CaPHM7 shows a filamentation defect, which might contribute to its reduced colony growth and attenuated virulence in the mouse model of systemic infection. Similar to the functions of other members of the anoctamin/TMEM16 family as sensors for various stresses including nutrient availability [7-13], the plasma membrane protein $\mathrm{CaPhm} 7$ might function to regulate zinc availability for $C$. albicans cells in media or within the host environment and thereby affect their filamentation and virulence.

\section{Conclusion}

In this study, we have demonstrated that $\mathrm{CaPhm} 7$ is localized to the plasma membrane in both yeast-form and filamentous cells of $\mathrm{C}$. albicans. $\mathrm{CaPhm} 7$ regulates ion homeostasis, drug tolerance, filamentation and virulence in this important human fungal pathogen. C. albicans cells lacking $\mathrm{CaPHM} 7$ have a defect in filamentation and attenuated virulence in the systemic mouse model. Therefore, CaPhm7 is a potential target for antifungal development.

\section{Additional file}

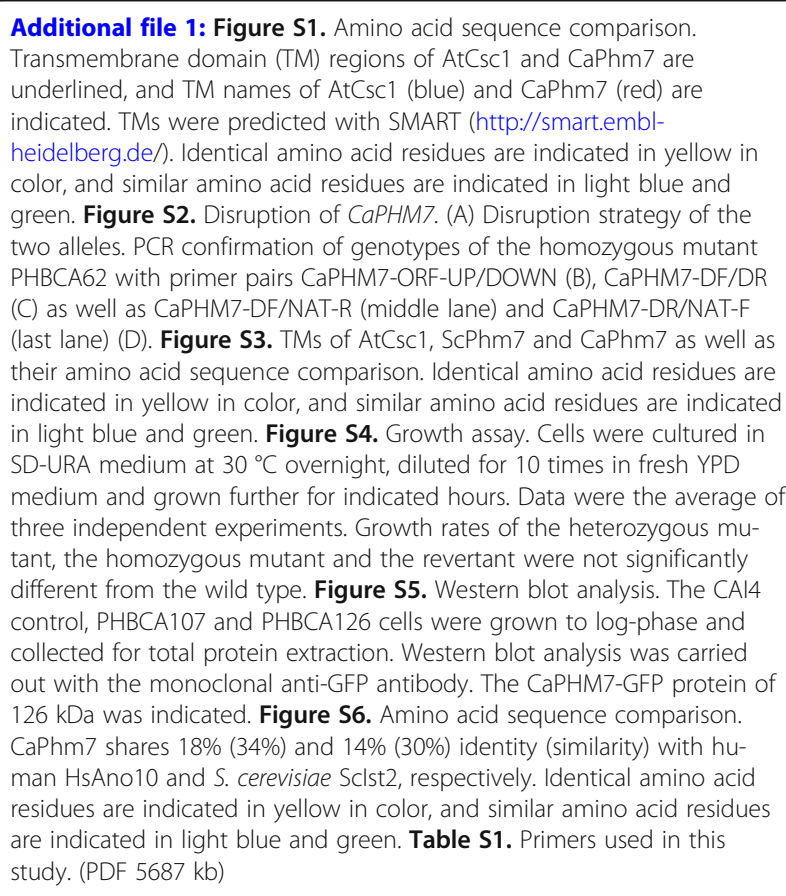

\section{Abbreviations}

CSC: Calcium permeable stress-gated cation channel; TRP: Calciumperméable transient receptor potential

\section{Acknowledgements}

We are grateful to Jianqing Cheng for his technical support.

\section{Funding}

This work is supported by the National Natural Science Foundation of China to $L$ (No. 81571966 and No. 81371784). The funding body plays no role in the design of the study and collection, analysis, and interpretation of data and in writing the manuscript. 


\section{Availability of data and materials}

The datasets used and/or analyzed during the current study are available from the corresponding author on reasonable request.

\section{Authors' contributions}

$\sqcup$ conceived and designed the study, analyzed the data as well as wrote the manuscript. HP performed the experiments. All authors have read and approved the manuscript.

\section{Ethics approval}

This study was submitted to and approved by the Ethics Committee of Nantong University, China, where we carried out our mouse experiments in this work, since there was no animal core facility at the Shandong University of Technology, China.

\section{Competing interests}

The authors declare that they have no competing interests.

\section{Publisher's Note}

Springer Nature remains neutral with regard to jurisdictional claims in published maps and institutional affiliations.

\section{Received: 5 February 2018 Accepted: 21 May 2018}

\section{Published online: 04 June 2018}

\section{References}

1. Plattner $\mathrm{H}$, Verkhratsky $\mathrm{A}$. The ancient roots of calcium signalling evolutionary tree. Cell Calcium. 2015:57:123-32.

2. Cui J, et al. Calcium homeostasis and signaling in yeast cells and cardiac myocytes. FEMS Yeast Res. 2009;9:1137-47.

3. Zhao Y, et al. The plasma membrane protein Rch1 is a negative regulator of cytosolic calcium homeostasis and positively regulated by the calcium/ calcineurin signalling pathway in budding yeast. Eur J Cell Biol. 2016;95:164-74.

4. Jiang $L$, et al. The Candida albicans plasma membrane protein Rch1p, a member of the vertebrate SLC10 carrier family, is a novel regulator of cytosolic $\mathrm{Ca}^{2+}$ homoeostasis. Biochem J. 2012:444:497-502.

5. Peiter $E$. The ever-closer union of signals: propagating waves of calcium and ROS are inextricably linked. Plant Physiol. 2016;172:3-4.

6. Swarbreck SM, Colaço R, Davies JM. Plant calcium-permeable channels Plant Physiol. 2013;163:514-22.

7. Yuan $\mathrm{F}$, et al. OSCA1 mediates osmotic-stress-evoked $\mathrm{Ca}^{2+}$ increases vital for osmosensing in Arabidopsis. Nature. 2014;514:367-71.

8. Hou C, et al. DUF221 proteins are a family of osmosensitive calcium permeable cation channels conserved across eukaryotes. Cell Res. 2014;24:632-5.

9. Chang $Y$, et al. Properties of the intracellular transient receptor potential (TRP) channel in yeast, Yvc1. FEBS Lett. 2010;584:2028-32.

10. Venkatachalam K, Montell C. Annu Rev Biochem. 2007:76:387-417.

11. Bonilla M, Cunningham KW. Calcium release and influx in yeast: TRPC and VGCC rule another kingdom. Sci STKE. 2002;2002:pe17.

12. Denis $\mathrm{V}$, Cyert MS. Internal $\mathrm{Ca}^{2+}$ release in yeast is triggered by hypertonic shock and mediated by a TRP channel homologue. J Cell Biol. 2002;156:29-34.

13. Harteneck C, Plant TD, Schultz G. From worm to man: three subfamilies of TRP channels. Trends Neurosci. 2000;23:159-66.

14. Picollo A, Malvezzi M, Accardi A. TMEM16 proteins: unknown structure and confusing functions. J Mol Biol. 2015;427:94-105.

15. Pedemonte N, Galietta LJ. Structure and function of TMEM16 proteins (aoctamins). Physiol Rev. 2014;94:419-59.

16. Williamson P. Phospholipid Scramblases. Lipid Insights. 2016:8:41-4.

17. Kunzelmann $\mathrm{K}$, et al. Modulating $\mathrm{Ca}^{2+}$ signals: a common theme for TMEM16, Ist2, and TMC. Pflugers Arch. 2016;468:475-90.

18. Feenstra $B$, et al. Common variants associated with general and MMR vaccine-related febrile seizures. Nat Genet. 2014:46:1274-82

19. Calderone RA, Fonzi WA. Virulence factors of Candida albicans. Trends Microbiol. 2001;9:327-35

20. Mayer FL, Wilson D, Hube B. Candida albicans pathogenicity mechanisms. Virulence. 2013:4:119-28

21. Feng J, et al. CaTip41 regulates protein phosphatase 2A activity, CaRad53 deactivation and the recovery of DNA damage-induced filamentation to yeast form in Candida albicans. FEMS Yeast Res. 2016;16 https://doi.org/10 1093/femsyr/fow009.
22. Wang $Y$, et al. Genetic interactions between the Golgi $\mathrm{Ca}^{2+} / \mathrm{H}^{+}$exchanger Gdt1 and the plasma membrane calcium channel Cch1/Mid1 in the regulation of calcium homeostasis, stress response and virulence in Candida albicans. FEMS Yeast Res. 2015;15(7).

23. Reuss O, Vik A, Kolter R, Morschhauser J. The SAT1 flipper, an optimized tool for gene disruption in Candida albicans. Gene. 2004;341:119-27.

24. Bachewich C, Nantel A, Whiteway M. Cell cycle arrest during S or M phase generates polarized growth via distinct signals in Candida albicans. Mol Microbiol. 2005:57:942-59.

25. Liu W, et al. The protein kinase CaSch9p is required for the cell growth, filamentation and virulence in the human fungal pathogen Candida albicans. FEMS Yeast Res. 2010;10:462-70.

26. Jiang $L_{\text {, }}$ et al. The putative $A B C$ transporter encoded by the orf19.4531 plays a role in the sensitivity of Candida albicans cells to azole antifungal drugs. FEMS Yeast Res. 2016;16 https://doi.org/10.1093/femsyr/fow024.

27. Lee K, Buckley HR, Campbell CC. An amino acid liquid synthetic medium for the development of mycelial and yeast forms of Candida albicans. Sabouraudia. 1975;13:148-53.

28. Gimeno CJ, Ljungdahl PO, Styles CA, Fink GR. Unipolar cell divisions in the yeast Saccharomyces cerevisiae lead to filamentous growth: regulation by starvation and RAS. Cell. 1992;68:1077-90.

29. Murad AM, et al. Clp10, an efficient and convenient integrating vector for Candida albicans. Yeast. 2000;16:325-7.

30. Brand $\mathrm{A}$, et al. Ectopic expression of URA3 can influence the virulence phenotypes and proteome of Candida albicans but can be overcome by targeted reintegration of URA3 at the RPS10 locus. Eukaryot Cell. 2004;3:900-9.

31. Li $X$, et al. The MAP kinase-activated protein kinase $R c k 2 p$ regulates cellular responses to cell wall stresses, filamentation and virulence in the human fungal pathogen Candida albicans. FEMS Yeast Res. 2010;10:441-51.

32. Xu D, et al. Genetic interactions between Rch1 and the high-affinity calcium influx system Cch1/Mid1/Ecm7 in the regulation of calcium homeostasis, drug tolerance, hyphal development and virulence in Candida albicans. FEMS Yeast Res. 2015:15:fov079.

33. Gow NA, et al. Candida albicans morphogenesis and host defence: discriminating invasion from colonization. Nat Rev Microbiol. 2011;10:112-22.

34. Heitman J. On the discovery of TOR as the target of rapamycin. PLoS Pathog. 2015:11:e1005245.

35. Ogawa N, DeRisi J, Brown PO. New components of a system for phosphate accumulation and polyphosphate metabolism in Saccharomyces cerevisiae revealed by genomic expression analysis. Mol Biol Cell. 2000;11:4309-21.

36. Serra-Cardona A, et al. Coregulated expression of the Nat/phosphate Pho89 transporter and Ena1 Na+-ATPase allows their functional coupling under high-pH stress. Mol Cell Biol. 2014;34:4420-35.

37. da Silva Ferreira ME, et al. Functional characterization of the Aspergillus fumigatus calcineurin. Fungal Genet Biol. 2007;44:219-30

38. Fonzi WA, Irwin MY. Isogenic strain construction and gene mapping in Candida albicans. Genetics. 1993;134:717-28

\section{Ready to submit your research? Choose BMC and benefit from:}

- fast, convenient online submission

- thorough peer review by experienced researchers in your field

- rapid publication on acceptance

- support for research data, including large and complex data types

- gold Open Access which fosters wider collaboration and increased citations

- maximum visibility for your research: over $100 \mathrm{M}$ website views per year

At BMC, research is always in progress.

Learn more biomedcentral.com/submissions 